\title{
On the performance of small-scale living facilities in nursing homes: A simulation approach
}

\author{
Dennis Moeke ${ }^{\mathrm{a}, \mathrm{c}, *}$, Ruben van de Geer ${ }^{\mathrm{b}}$, Ger Koole ${ }^{\mathrm{b}}$, Rene Bekker ${ }^{\mathrm{b}}$ \\ ${ }^{\text {a }}$ Faculty of Economics and Business Administration, VU University Amsterdam, The Netherlands \\ ${ }^{\mathrm{b}}$ Department of Mathematics, VU University Amsterdam, The Netherlands \\ ${ }^{\mathrm{c}}$ Research Group Logistics and Alliances, HAN University of Applied Sciences, The Netherlands
}

\section{A R T I C L E I N F O}

\section{Article history:}

Received 3 December 2015

Accepted 12 October 2016

Available online 25 October 2016

\section{Keywords:}

Nursing home care

Simulation

Small-scale living facilities

Staffing

Client-centered care

\begin{abstract}
A B S T R A C T
Scientific evidence on the impact of small-scale living facilities (SSLFs) on quality of life of nursing home clients remains scarce. In this study a simulation model is developed to examine the performance of SSLFs, in terms of meeting the time preferences of their residents. We model scheduled care using historical data and unscheduled care using a Poisson-Gamma mixture model. The model is used to explore the impact of a change in demand characteristics, duration of care delivery, travel time, allocation flexibility, shifts, number of clients and allocation policy. The results show that to further improve the performance, the focus should lie on: (1) increasing the allocation flexibility of care workers and the number of clients per SSLF, and (2) time dependent staffing. Furthermore, this study shows that simulation is a useful tool for assessing and improving daily nursing home operations. The presented simulation model provides a basis for building a decision support tool for nursing home managers.
\end{abstract}

(C) 2016 Elsevier Ltd. All rights reserved.

\section{Introduction}

In the last decades, many Dutch nursing homes have transformed their care environments into more client-centered, smallscale care settings. These small-scale settings, often referred to as small-scale living facilities (SSLFs) or small-scale care facilities, provide care and/or support to a small group of residents in a homelike environment. This approach aims to enable residents to live their lives according to their own needs and preferences [1] or as stated by [2, p. 804] "daily life and care provision are adjusted to residents lifestyle and preferences as much as possible". Despite the fact that most nursing homes have embraced the concept of SSLFs as a way to give substance to client-centered care, scientific evidence on the impact of SSLFs on quality of life $(\mathrm{QOL})$ is still scarce $[3,4]$.

In order to make it possible for nursing home residents to live their lives according to their needs and preferences, the necessary care and support should be delivered as close as possible to the time preferences of the residents. Hence, with regard to the delivery of care and support, earliness and waiting should be

\footnotetext{
* Correspondence to: Amsterdam Center for Business Analytics (ACBA), VU University Amsterdam, De Boelelaan 1105, 1081 HV Amsterdam, The Netherlands.

E-mail address: d.moeke@vu.nl (D. Moeke).
}

avoided, without overstretching the available budget. From this perspective, QoL largely depends on the co-ordination and timing of service delivery.

In this study a simulation model is developed to examine the performance of SSLFs, in terms of meeting the time preferences of their residents, under different assumptions. Furthermore, improvements in the allocation of care workers are suggested. Simulation is a commonly used method to study the effectiveness or efficiency of larger and/or complex systems which do not lend themselves for analytic approaches. Regarding the purpose of this study, the main advantage of a simulation approach is its flexibility, as parameters and assumptions of the underlying model can be adjusted relatively easy. As such, simulation (1) is well suited to answer "what-if" questions and (2) allows for a detailed analysis of how components interact and of the trade-offs involved.

Several studies [5-8] show that, during the past two decades, simulation has been extensively used for modeling healthcare systems. The vast majority of existing studies focus on supporting better operational decision-making and planning in a hospital setting, with an emphasis on specific subsystems. Examples of those subsystems are: operating theaters $[9,10]$, emergency departments $[11,12]$ and intensive care units $[13,14]$. As mentioned by Van Eeden et al. [15], to date the area of nursing home care received hardly any attention in the Operations Research (OR) literature. To the best of our knowledge this is the first study examining the daily operations of SSLFs. 
The remainder of this paper is structured as follows. In the next section, the study is outlined and justified by providing an overview of the existing literature. Then, in Section 3 we present the analysis of the input data and in Section 4 the model is described. The scenarios together with their performance are presented in Section 5. In Section 6, for each half-hour interval, we determine the number of care workers required to meet a predetermined service level. Finally, in the last section, conclusions are drawn and the results are discussed.

\section{Background}

Today, the concept of SSLFs is applied in both somatic and psychogeriatric care settings. Most research on the effects of SSLFs has been conducted in psychogeriatric settings. This is not surprising considering the fact that SSLFs were originally developed for residents suffering from dementia. The term 'dementia' is a catch-all term for a group of symptoms caused by gradual death of brain cells [16]. Common symptoms are problems with concentration, memory, thinking, behavior and the ability to perform everyday activities. The often progressive nature of the disease makes that carrying out activities of daily living (ADL) becomes more and more of a challenge and causes an increase in dependency. Therefore, as the disease progresses, institutional nursing is often inevitable. Until the eighties of the last century the behavior of dementia clients was mainly interpreted from a disease perspective, whereby little attention was paid to the experiences and perceptions of the dementing person [17]. From the nineteen nineties onwards, more and more emphasis was put on how people with dementia cope with the consequences of their illness and on how they experience and value their personal situation [18]. The aim to make dementia care fit the feelings and emotional needs of each individual client has led to development of so-called 'integrated emotion-oriented care', in which "the offered care and activities are person-centered and well attuned to the abilities, experiences and preferences of the person with dementia" [19, p. 153]. This shift towards more holistic dementia care was accompanied by the emergence of small-scale and homelike nursing home settings. In the Netherlands, the first SSLF was introduced in the early nineteen eighties. In the decades that followed, encouraged by government policies and programs, the number of SSLFs increased. In 2010, roughly 25\% of the residents with dementia who received nursing home care lived in SSLFs [20]. Despite the lack of a uniform definition of a SSLF, the following common characteristics regarding SSLFs can be identified [1]:

1. Providing care and/or support to $6-8$ residents.

2. Home-like environment.

3. Small fixed team of care workers.

4. Care workers perform a broad range of tasks.

5. More individual decision making by care workers.

6 . Residents should have influence on their daily routine.

Today it is generally assumed that living in a SSLF will add to the QoL, however scientific evidence to support this assumption is sparse and mixed in its results.

In a comparative study, Te Boekhorst et al. [21] examined the effects of small-scale living for people with dementia compared with living in a traditional nursing home setting. The results of this study show that the residents living in a SSLF (1) needed less assistance with ADLs, (2) had more social engagement, (3) had greater sense of aesthetics, (4) had more to do during the day and (5) were prescribed less physical restraints. However, the authors did not find differences in cognitive status, behavioral problems and the prescription of psychotropic drugs. Verbeek et al. [22] also investigated the effects of small-scale living compared with traditional nursing home care. The purpose of that study was to evaluate the effects of small-scale living facilities in dementia care on residents ( $Q \mathrm{LL}$ and behavior), family caregivers (experienced burden, involvement with care and satisfaction), and staff (job satisfaction and motivation). This study found no effect on residents' total QoL. However, family caregivers in SSLFs experienced less burden and were more satisfied with the nursing staff compared to family caregivers in traditional nursing homes. With regard to the involvement of family caregivers, no significant differences were found. Also, no significant differences were found for staffs' job satisfaction and motivation. Given these mixed results, the authors concluded that providing small-scale care "may not be a final solution to accomplish high-quality dementia care and that other options should be considered" [22, p. 662].

Using a quasi-experiment, De Rooij et al. [3] examined the benefits of small-scale living for residents with dementia, compared to traditional long-term care in the Netherlands and Belgium. Their findings indicate that both small-scale and traditional settings appear to have beneficial effects on different domains. The Dutch sample showed higher scores on 'social relations', 'positive affect' and 'having something to do' for small-scale settings compared to residents in traditional settings. Moreover, mean scores on 'caregiver relation' and 'negative affect' remained more stable over time among residents in small-scale settings compared to traditional settings. In the Belgian sample, the differences found between traditional and small-scale settings were less evident. The authors also found that residents 'felt more at home' in traditional settings. Moreover, the mean QoL scores on 'restless behavior', 'having something to do' and 'social relations' remained stable in the traditional setting but decreased in the small-scale settings.

The recent studies of Moeke et al. [23] and Lieder et al. [24] show that, when it comes to efficiently meeting the time preferences of nursing home clients, scale of scheduling plays a prominent role. Applying small-scale scheduling can lead to less efficiency in terms of number of care workers needed to meet the demand. We believe the disadvantages of organizing care on a small scale can be compensated for by applying job enlargement, but only up to a certain extent. Hence, our preliminary hypothesis is that when the scale of scheduling becomes too small, it will become impossible to meet the preferences of the nursing home residents (in terms of moment and time) without increasing the number of care workers. As care and support activities in SSLFs are organized on a relatively small scale, we expect the quality of care in terms of meeting the time preferences of the residents to be low (i.e., we expect waiting times to be relatively long).

The main contributions of this study can be summarized as follows. Using simulation and real-life data we:

1. Examine the performance of SSLFs, in terms of meeting the time preferences of their residents, under different assumptions.

2. Suggest improvements (i.e., alternative approaches) regarding the allocation of care workers, using the simulation results.

3. Show that simulation can be a useful tool for assessing and improving daily nursing home operations.

\section{Preliminary data analysis}

For the delivery of care and support in a nursing home, two types of care activities can be distinguished. For some of the care activities it is possible, based on the needs and preferences of the clients, to make a fairly detailed schedule in advance. Examples of this type of activities are 'giving medicine' and 'help with getting out of bed in the morning'. These activities are also referred to as 'care by appointment' or 'scheduled care' [23,24]. On the other hand, there are healthcare activities which are carried out in 
response to random or unexpected demand such as assistance with toileting. Activities of this type are also referred to as "care on demand' or 'unscheduled care' [15]. In this study we focus on the care process during daytime (7:00-23:00 h). During the night care requests are mainly unscheduled and the main challenge is to set appropriate staffing levels for swift response. This issue has been addressed in [15]. During the day, the mixture of unscheduled and scheduled care provide new challenges that have not been addressed until now. Therefore, this is the focus of the current paper.

The main emphasis of this section is on the analysis of the main characteristics of the unscheduled care process. In order to make assumptions regarding the demand characteristics and service times of scheduled healthcare tasks, we used the analysis presented in [25] and the data that were collected for this study.

\subsection{Scheduled care}

For unscheduled care, we use the demand patterns observed in [25]. Specifically, in [25] the preferred activity times, so called PAT's, have been analyzed for 5 different departments of a Dutch nursing home (see Table A.12 for some characteristics of the departments). The results presented in [25] indicate that the PATs hardly vary between days within each department. Fig. 1 shows the relative PAT-values in relation to the total number of clients during the course of the day for all departments together. The plot shows there is considerable fluctuation in the number of PATs over the course of a day. Most of the fluctuation can be explained by the major activities of daily living. Between 0:00 and 6:00 there are hardly any activities (some clients have medication though). During the early morning, most residents need help with getting out of bed and/or dressing. Around lunch and dinner time, there is need for assistance with feeding. Finally, at the end of the day, some of the residents need assistance with getting to bed. The average care duration for scheduled care is $12.64 \mathrm{~min}$; see Fig. A.12 for the variation in expected care delivery times.

\subsection{Unscheduled care}

To make assumptions about the demand and service times of unscheduled healthcare tasks, ${ }^{1}$ we use the results presented in [15] and the data that were collected for this study. From [15] it can be concluded that the unscheduled care requests during the night is well approximated by a homogeneous Poisson process. Since Poisson processes naturally appear in the modeling of completely random phenomena, it might be tempting to use a homogeneous Poisson process for unscheduled care during the day as well. However, the boxplots in Fig. 2 indicates that the arrival process during daytime might be time dependent. To model this, we look for a practical option that is line with the intuition of practitioners.

In the data, we exclude lunch and dinner times (11:30-12:30 and 16:30-17:30 $\mathrm{h}$ ) as care is often delivered in a different manner, e.g. meals are consumed in shared room. In Fig. 2, we also see that the number of unscheduled care requests is very small. Now, to confirm statistically whether or not the arrival process is constant during the day, we use Pearson's $\chi^{2}$-test [26]. For each of the 90 days, the $\chi^{2}$-test is used to determine whether the empirical arrival frequencies originate from a Poisson distribution. We use the following property of the Poisson process: given a fixed number of arrivals in an interval, the numbers of arrivals in nonoverlapping subintervals have jointly a multinomial distribution.

\footnotetext{
1 It should be noted that in this chapter an unscheduled care request is also referred to as a PAT.
}

Table 1

Variance-to-mean ratio for each time period.

\begin{tabular}{llll}
\hline Time period & Average \# call requests & Variance & Variance-to-mean \\
\hline 7:00-12:00 & 46.00 & 69.19 & 1.50 \\
12:00-18:00 & 47.04 & 125.59 & 2.67 \\
18:00-23:00 & 59.87 & 118.57 & 1.98 \\
\hline
\end{tabular}

If the Poisson process is homogeneous between 7:00 and 23:00 $\mathrm{h}$ and the subintervals are of an equal length of $30 \mathrm{~min}$ (i.e., 28 subintervals), then the multinomial probabilities would be equal (i.e., 1/28). The $\chi^{2}$-test is performed in order to compare empirical arrival frequencies with the multinomial distribution. Using a significance level of 0.05 , the null hypothesis is rejected for 18 (20\%) of the 90 days.

Based on the test results we assume the arrival process to be time dependent. To account for this time dependency, we divide the day into three time periods: (1) morning (7:00-12:00 h), (2) afternoon (12:00-18:00 h) and evening (18:00-23:00 h) as these time periods coincide with the repetitive cycle of daily routines. Next we repeat the $\chi^{2}$-test for each of the time periods separately. ${ }^{2}$ The null hypothesis is now rejected for $13 \%$ (morning), $14 \%$ (afternoon) and 6\% (evening) of the 90 days. Hence, we see that the arrival process yields a more stable pattern. We note that it would be possible to distinguish different hourly arrival rates; we chose not to do this for ease of presenting the model to practitioners. Moreover, the simulation results later indicate that the precise arrival pattern of unscheduled care has limited impact.

For the three time periods (morning, afternoon, and evening), we calculated the mean and variance of the number of unscheduled care requests per time period, see Table 1 . The value-to-mean ratio (also presented in Table 1) is clearly larger than 1. For a Poisson process the value-to-mean ratio should be equal to 1 . Hence, the amount of variability in the number of unscheduled care request is larger than you would obtain from a Poisson process; this is also commonly referred to as overdispersion.

A substantial part of the observed overdispersion is presumably attributable to a small number of frequent callers. From the data it can be seen that for only $22 \%$ of the clients the average number of call requests per day is more than 1 . In addition, for most of these frequent callers, the total number of calls fluctuates considerably from day to day; this is illustrated in Fig. A.13 for four frequent callers. One possible explanation of the observed spikes in the number of requests is temporarily poor health conditions.

\section{Simulation model}

We consider a nursing home department consisting of 4 SSLFs. Each SSLF provides $24 / 7$ care and support to six psychogeriatric clients in a small archetypical house-like facility. The nursing home department aims to deliver the necessary care and support as close as possible to the time preferences of the residents. In this section, we discuss the elements of the simulation model, which is developed in Python 2.7.9. In the simulation model, we simulate the operation during daytime, i.e., 7:00-23:00, for 2000 days (see Section 4.5). As discussed in Section 3 the night is covered in [15] and is outside the scope of the current paper. For each day, we do the following:

- Scheduled care is generated using historic PAT data, see Section 4.1.

- Unscheduled care is generated using a Poisson-Gamma mixture model, see Section 4.2 .

- Assignment of staff to care requests is by default First-come first-served (FCFS), see Section 4.3.

- The performance indicators, involving waiting times and occupancy are determined, see Section 4.4.

\footnotetext{
2 Lunch and dinner time are again excluded from the analysis.
} 


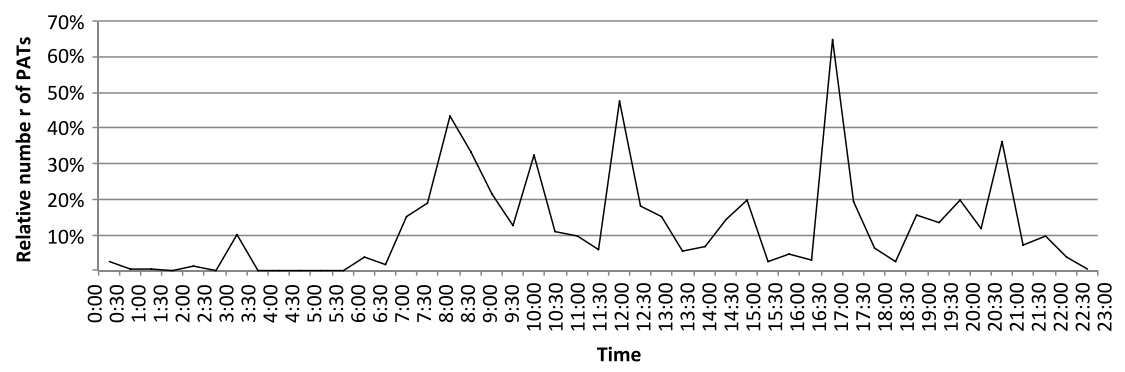

Fig. 1. Relative PAT-values in relation to the total number of clients during the day.

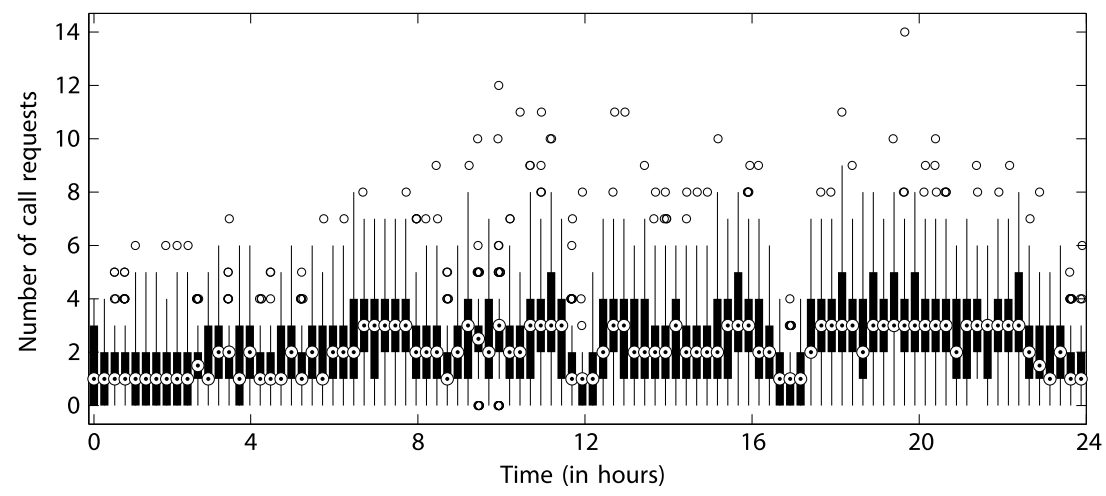

Fig. 2. Boxplots of the number of call requests per quarter hour.

\subsection{Scheduled care}

For the purpose of this simulation study, for each simulation round, the SSLFs are 'filled' with new clients. Together with their PATs for scheduled care and corresponding expected service times, each client is randomly chosen from the empirical datasets of the five departments (see Table A.12). As such, we assume there are no differences between psychogeriatric and somatic clients in terms of PATs and the corresponding expected service times. We believe this assumption is plausible because scheduled care mainly consists of providing help and support with ADLs and there is no considerable difference (in terms of number and duration) between psychogeriatric and somatic clients with respect to these activities.

\subsection{Unscheduled care}

The test results presented in Section 3 show that the arrival rate of unscheduled care depends on the time of the day. In order to account for this time-dependency, for each time period defined in Section 3 we calculate the ratio of the real average number of request during the time period to the average number of request when the total number of requests would have been evenly spread throughout the day (i.e., the expected number of arrivals). We refer to this ratio as the time period index (TPI). The TPIs for the morning, afternoon and evening are respectively $0.94,0.96$ and 1.10 . We let $\lambda$ represent the arrival rate (requests per hour). Using the TPIs, the average number of requests per hour for the three time periods would then be set as follows: $0.94 \lambda, 0.96 \lambda$ and $1.10 \lambda$. Based on observations in three different nursing homes, we estimate that in practice the average number of request for unscheduled care is 3 per hour per SSLF. However, as detailed insight into the number of request for the setting under study is lacking, the effect of different arrival rates will be examined (see Section 5).

To account for the observed additional variability in demand (i.e., overdispersion) we model the average number of requests as a random variable using a Poisson-Gamma mixture model (see e.g., [27]). Hence, we assume the demand for unscheduled
Table 2

Time period index.

\begin{tabular}{llll}
\hline Time period & Average \# arrivals & Expected \# arrivals & TPI \\
\hline 7:00-12:00 & 46.00 & 49.15 & 0.94 \\
12:00-18:00 & 47.04 & 49.15 & 0.96 \\
18:00-23:00 & 59.87 & 59.87 & 1.10 \\
\hline
\end{tabular}

care follows a Poisson process with random rate $\Lambda_{t}$, where $\Lambda_{t} \sim \operatorname{Gamma}\left(\alpha_{t}, \beta_{t}\right)$. The number of arrivals in this mixture model follows a negative binomial distribution with parameters $\alpha_{t}$ and $\frac{1}{1+\beta_{t}}$. The subscript $t$ emphasizes the dependence on the time period of the day (e.g., morning, afternoon and evening). See e.g., [28] for a derivation of the negative binomial as a Poisson-Gamma mixture.

It remains to estimate parameters values $\alpha_{t}$ and $\beta_{t}$. Define

$A_{t}=$ estimated average number of arrivals during time period $t$

$I_{t}=$ observed rate of dispersion during time period $t$.

To determine $\alpha_{t}$ and $\beta_{t}$, we solve the following set of linear equations

$\left\{\begin{array}{l}\frac{\alpha_{t}}{\beta_{t}}=A_{t} \\ \frac{1}{\beta_{t}}=I_{t}\end{array} \Leftrightarrow\left\{\begin{array}{l}\alpha_{t}=\frac{A_{t}}{I_{t}} \\ \beta_{t}=\frac{1}{I_{t}} .\end{array}\right.\right.$

The first equation ensures that the average number of Poisson arrivals equals the number of arrivals observed and the second equation sets the appropriate rate of dispersion.

Next, the negative binomial distribution is fitted to the data. To assess the goodness-of-fit of the negative binomial distribution on the data we conduct a $\chi^{2}$-test. We prefer the $\chi^{2}$-test over the Kolmogorov-Smirnov (KS) test, which is arguably the most popular non-parametric goodness-of-fit test available, since the KS test is not valid when the distribution's parameters must be estimated from the sample or when the null distribution is discrete [29]. The test results are presented in Table 3. The found $p$-values indicate that there is no evidence against the null 
Table 3

Test results fitting the negative binomial distribution.

\begin{tabular}{|c|c|c|c|c|c|c|}
\hline & \multirow{2}{*}{$\chi^{2}$-statistic } & \multirow[t]{2}{*}{$p$-value } & \multirow[t]{2}{*}{ \# Bins } & \multirow[t]{2}{*}{ Bin size } & \multicolumn{2}{|c|}{ Parameters } \\
\hline & & & & & $\overline{\hat{r}}$ & $\hat{p}$ \\
\hline Morning & 5.22 & 0.27 & 7 & 3 & 27 & 0.27 \\
\hline Afternoon & 6.15 & 0.29 & 8 & 3 & 15 & 0.40 \\
\hline Evening & 9.78 & 0.13 & 9 & 3 & 41 & 0.23 \\
\hline
\end{tabular}

hypothesis that the number of arrivals follows a negative binomial distribution. In addition, Figs. A.14-A.16 visually show a good fit. Based on these results we assume the number of arrivals follow a Poisson-Gamma mixture.

When it comes to the delivery times of unscheduled healthcare tasks, the analysis presented in [15] indicate that the hyperexponential distribution gives the best fit with the data. Furthermore, in practice there is always a certain amount of time between a request of a client and the moment a care worker is present, referred to a travel time. We model traveling time by incorporating a deterministic travel time between two successive tasks within the same SSLF and between two SSLFs.

\subsection{Staffing and task assignment}

In line with the common characteristics of SSLFs mentioned in Section 2 we assume each care worker has the required qualifications to perform all tasks. Furthermore, a care worker can be assigned to either a single or to multiple SSLFs. With respect to the assignment of tasks the following strategies are distinguished:

(1) FCFS strategy-The provision of care and support is on FCFS basis. In this fully reactive approach, no distinction is made between scheduled and unscheduled care. The FCFS procedure can be described as follows:

- Each day is divided into time buckets of $1 \mathrm{~min}(t=1,2, \ldots$, 960).

- Starting at time 7:00 $(t=1)$, for every time step, healthcare tasks based on PATs are assigned to the first available care workers.

- When a client has a care request during the handling of an earlier PAT (i.e., an additional PAT), this additional request will be adhered to by the same care worker. The waiting time for this additional PAT is set to 0 .

- When all care workers are busy, a virtual queue is filled with the remaining activities.

- PATs in the queue have priority over a 'new' PAT.

- When a care worker is providing care during change of shifts, he or she will first complete the task.

(2) Task scheduling-In this approach scheduled and unscheduled care activities are organized separately. Using the OERplanner algorithm (for more detailed info see [23]), scheduled healthcare tasks are planned (if possible) within 15 min from the time preference of the client. In addition, for unscheduled healthcare care tasks (i.e., unscheduled care), the FCFS approach is used.

\subsection{Performance measures}

To assess the performance of the scenarios presented in the next section, for each simulation run, we calculate the following performance measures:

- Average waiting time at time $t$-The average time a task has spent in the queue (for all tasks in the queue) at time $t$.

- Total average waiting time-The average waiting time over all $t$.

- Queue length at time $t$-The number of tasks waiting in the queue at time $t$.
- 15 min service level in time-interval $\Delta t$-The \% of care requests which originated in time-interval $\Delta t$ for which a care worker was present with the client within 15 min. $^{3}$

- Overall service level-The average service level over all $\Delta t$.

- Occupancy rate at time $t$-The number of care workers handling care requests at time $t$ divided by the total number of care workers present at time $t$.

- Total average occupancy rate-The average occupancy rate over all $t$.

Subsequently, the performance measures are averaged over all simulation runs.

\subsection{Number of simulation runs and validation}

Because of the random characteristics of the simulation model a certain number of runs is needed in order to obtain sufficiently accurate results. To determine the required number of simulation runs, for each minute between 7:00 and 23:00, we examine the attained confidence intervals progressively throughout the simulation. We continue to iterate until the predefined confidence interval has converged to an acceptable level of accuracy. This approach is referred to by [31] as the graphical method. Using a $99 \%$ confidence level, and a maximum acceptable confidence interval width of 2 min, it was found that a minimum number of 2000 simulation runs is required. Fig. 3 shows the average waiting time during the course of the day for the base case over 2000 runs (the black line) and the corresponding confidence intervals (red dotted line).

The simulation model was validated by (1) manually checking the logs for inconsistencies and (2) comparing the results of the simulation with the OERplanner [23] outcomes for scheduled care and the Erlang-delay model (see e.g., [32]) outcomes for unscheduled care.

\section{Scenarios}

In the next sections we present the simulation results of the base case (presented in Section 5.1) and explore the impact of a change in:

1. Demand for unscheduled care

2. Duration of care delivery for unscheduled care

3. Travel time

4. Allocation flexibility

5. Number of clients

6. Allocation policy.

\subsection{Base case}

The base case, which resembles a real-life SSLF-setting, can be described as follows. We consider a Dutch nursing home department consisting of four SSLFs. Each of the four SSLFs provides 24/7 care and support to six psychogeriatric clients in a small archetypical house-like facility. In the Netherlands the type and amount of care needed by a client is based upon a Care Intensity Package score (ZZP score). Each package is assigned a maximum tariff set by the Dutch Health Care Authority. On average, the clients in the department under study have an indication for ZZP 7, which can be described as a need for 'sheltered living with very intensive caring due to specific disorders with an emphasis upon guidance'.

\footnotetext{
3 In the Netherlands, a 15 min target is regularly used when it comes to the provision of ADL care (see e.g., [23,30]).
} 

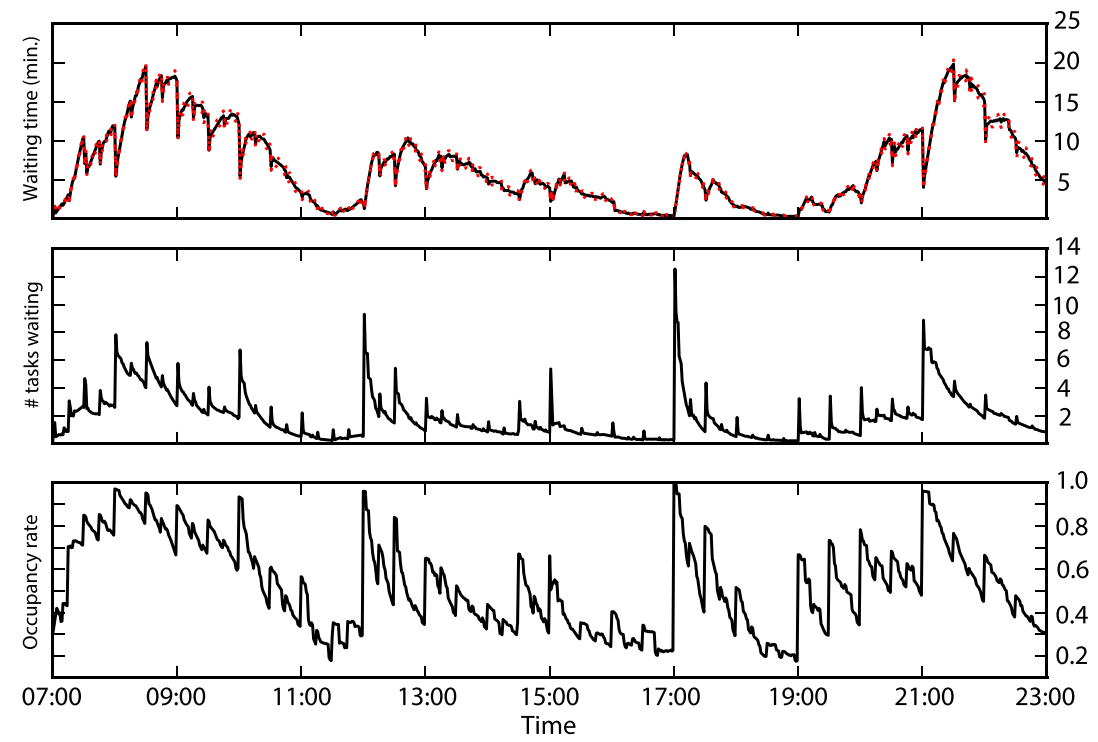

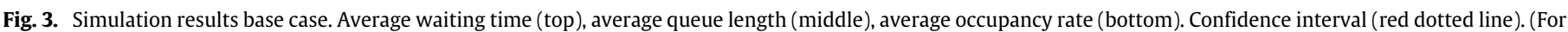
interpretation of the references to color in this figure legend, the reader is referred to the web version of this article.)

Table 4

Shifts base case.

\begin{tabular}{lllll}
\hline Shift & Time & Number of hours & Number of shifts & Total per 24 hours \\
\hline Day & $7: 00-15: 00$ & 8 & 4 & 32 \\
Morning & $7: 30-11: 30$ & 4 & 2 & 8 \\
Afternoon/evening & $15: 00-23: 00$ & 8 & 4 & 32 \\
Evening assistance & $16: 00-20: 00$ & 4 & 1 & 4 \\
Total & & & & 76 \\
Hours/client & & & & 3.17 \\
\hline
\end{tabular}

During daytime (7:00-23:00 $\mathrm{h}$ ) each SSLF has one fixed care worker. In addition, from 7:30 to 11:30 h two more care workers are available. These care workers are not dedicated to a single SSLF, but each provide support to two SSLFs (i.e., flexible care workers). From 16:00 to 20:00 h one flexible care worker is available who provides support to all four SSLFs. Details on the shifts are given in Table 4. The provision of care and support services in each of the SSLFs is on FCFS basis. Hence, in the base case setting, no distinction is made between scheduled and unscheduled services. When it comes to unscheduled care, we assume the arrivals to be negative binomial distributed and the average number of requests to be 3 per hour per SSLFs. Using the TPIs of Table 2, the average number of requests per hour for the three time periods is set as follows: 2.8 (morning), 2.9 (afternoon) and 3.3 (evening).

Based on the findings presented in [15] we assume the care delivery times to be hyperexponentially distributed with $\hat{p}_{1}=$ $0.10, \hat{p}_{2}=0.90, \hat{\mu}_{1}=0.11, \hat{\mu}_{2}=0.56$. These parameters can be interpreted as follows: with probability $\hat{p}_{2}=0.90$, the client has a minor request taking on average $1 / \hat{\mu}_{2}=1.79 \mathrm{~min}$, whereas with probability $\hat{p}_{1}=0.10$ the client has a large request taking $1 / \hat{\mu}_{1}=9.28 \mathrm{~min}$ on average. Hence, the expected care delivery time is $2.56 \mathrm{~min}$. In the base case we assume the travel time to be one minute between two successive tasks within the same SSLF and two minutes between two SSLFs.

The simulation results of the base case are presented in Fig. 3 and Table A.13. The results show that the total average waiting time per task is $5.73 \mathrm{~min}$. Furthermore, $88.35 \%$ of the healthcare tasks are provided within 15 min of the preferred delivery time. The total share of unscheduled tasks is 55\% i.e., the total share of scheduled task is $45 \%$. Although the average waiting time is less than 6 min, from Fig. 3 (top) it can be seen that the average waiting time fluctuates considerably over the course of a day, with average waiting times up to $20 \mathrm{~min}$ around 8:30 and 22:00 h. Most of the observed spikes in average waiting time can be explained by major activities in daily living (see also Section 3.1.). Despite the fact that two additional care workers are available during the morning, the occupancy rate (i.e., the workload) remains high during this period of the day (see Fig. 3 (bottom)). This notion is supported by the findings presented in [23]. The total average occupancy rate is $52.81 \%$.

\subsection{Scenario 1: demand for unscheduled care}

In this scenario we examine the effects of changes in the assumptions on the arrival process of unscheduled care.

First, we look at the effect of changing the parameters under time-dependent negative binomial assumptions (i.e., the base case assumption). The top part of Table 5 provides an overview of the configurations. From the results (see Fig. 4 and Table A.13) it can be seen that an increase in the arrival rate of unscheduled care has only a moderate impact on the overall service level. Hence, an increase in the average arrival rate from $3 / \mathrm{hr}$ to $6 / \mathrm{hr}$ results in a decrease in service level of only $3.87 \%$. An explanation for this observation would be that, compared to scheduled care, the demand for unscheduled care is more evenly spread throughout the day. Therefore, an increase in demand for unscheduled care puts relatively little additional pressure on the busy periods during the day. Furthermore, the average service time of unscheduled healthcare tasks is considerably less that of scheduled healthcare tasks. This explanation seems plausible as Scenario 2 (see Fig. 5) shows that an increase in the average care duration has substantial impact on the overall service level. Furthermore, compared to the base case, Scenario 1c shows a 1.55 min longer total average waiting time. Finally, the results presented in Table A.13 show that an increase in the arrival rate of unscheduled care has a substantial impact on the total average occupancy rate. 


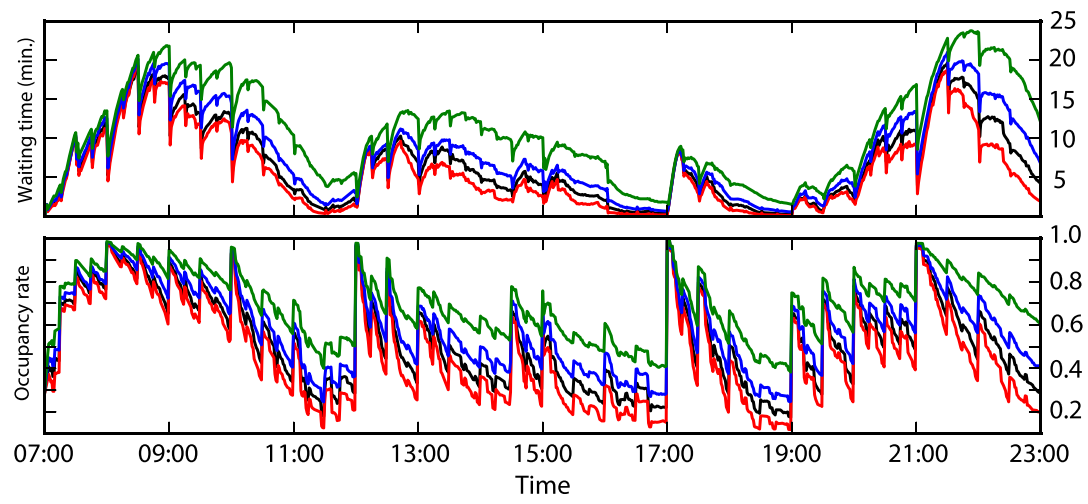

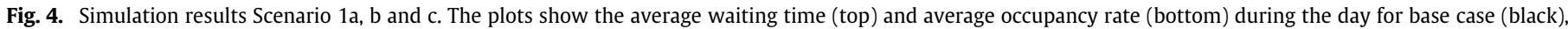
Scenario 1a (red), 1b (blue) and 1c (green). (For interpretation of the references to color in this figure legend, the reader is referred to the web version of this article.)

Table 5

Configurations Scenario 1.

\begin{tabular}{|c|c|c|c|c|}
\hline \multicolumn{5}{|c|}{ Time-dependent neg. binomial arrivals } \\
\hline \multirow[t]{2}{*}{ Scenario } & \multicolumn{4}{|l|}{ Rate } \\
\hline & Average & Morning & Afternoon & Evening \\
\hline Base case & $3 / \mathrm{hr}$ & $2.8 / \mathrm{hr}$ & $2.9 / \mathrm{hr}$ & $3.3 / \mathrm{hr}$ \\
\hline 1a & $2 / \mathrm{hr}$ & $1.9 / \mathrm{hr}$ & $1.9 / \mathrm{hr}$ & $2.2 / \mathrm{hr}$ \\
\hline $1 b$ & $4 / \mathrm{hr}$ & $3.7 / \mathrm{hr}$ & $3.8 / \mathrm{hr}$ & $4.4 / \mathrm{hr}$ \\
\hline \multirow[t]{7}{*}{$1 \mathrm{c}$} & $6 / \mathrm{hr}$ & $5.6 / \mathrm{hr}$ & $5.7 / \mathrm{hr}$ & $6.6 / \mathrm{hr}$ \\
\hline & \multicolumn{4}{|c|}{ Homogeneous Poisson arrivals } \\
\hline & Scenario & & Rate & \\
\hline & $1 d$ & & $2 / \mathrm{hr}$ & \\
\hline & $1 \mathrm{e}$ & & $3 / \mathrm{hr}$ & \\
\hline & 1f & & $4 / \mathrm{hr}$ & \\
\hline & $1 \mathrm{~g}$ & & $6 / \mathrm{hr}$ & \\
\hline
\end{tabular}

Table 6

Configurations Scenario 2.

\begin{tabular}{lll}
\hline Scenario & Distribution & Average care duration in minutes \\
\hline Base case & Hyperexponential & 2.56 \\
$2 \mathrm{a}$ & Exponential & 3 \\
2b & Exponential & 4 \\
$2 \mathrm{c}$ & Exponential & 6 \\
$2 \mathrm{~d}$ & Exponential & 8 \\
\hline
\end{tabular}

Next, we examine the performance under the assumption of time-homogeneous Poisson arrivals. The bottom part of Table 5 shows the configurations. As can be seen from the results presented in Table A.13, the performance differs only slightly from the performance under negative binomial assumptions. Hence, when it comes to the arrival process, the overall service level and total average waiting time are relatively insensitive to the assumptions explored.

\subsection{Scenario 2: duration of care delivery for unscheduled care}

In this scenario we assess the effect of less fluctuation in the care delivery times for unscheduled care by assuming exponentially distributed care delivery times. In addition, we examine the impact of an increase in the care duration for unscheduled care activities. The different configurations are presented in Table 6 .

The results (see Fig. 5 and Table A.13) show that the performance of Scenario 2a closely resembles the performance obtained for the base case. Furthermore, a change in the average care duration for unscheduled care from 3 to 8 min results in an increase of the total average waiting time of almost $6 \mathrm{~min}$. The 15 min service level drops from $88.04 \%$ to $77.69 \%$. Hence, it can be concluded that the overall performance is moderately sensitive to changes in the average care duration of unscheduled care.

\subsection{Scenario 3: travel time}

To assess the impact of travel time on the simulation results, multiple configurations are examined (see Table 7). We vary both the travel time within and between SSLFs. The travel times remain deterministic.

Figs. 6 and 7 show that the performance is more sensitive to a change in travel time within SSLFs than between SSLFs. This can be explained by the fact that most of the care workers are allocated to a specific SSLF. Consequently, a change in travel time between two successive tasks within the same SSLF has a relative large impact on the overall performance (see Table A.13).

\subsection{Scenario 4: allocation flexibility}

In this scenario we examine the impact of an increase in the allocation flexibility of care workers. Table 8 shows the examined configurations and Fig. 8 the corresponding results. It can be observed that especially an increase in allocation flexibility of fixed care workers has a substantial positive effect on the overall performance. Making fixed care workers fully flexible, results in an total average waiting time of $28.44 \%$ below the base case and a $6.72 \%$ improvement in service level. This increase in the overall performance due to more flexibility is in line with the findings presented in [23].

Table 7

Configurations Scenario 3.

\begin{tabular}{llll}
\hline Scenario & Travel time in minutes within a SSLF & Scenario & Travel time in minutes between SSLFs \\
\hline Base case & 1 & & 2 \\
3a & 0 & $3 \mathrm{e}$ & 0 \\
3b & 2 & $3 \mathrm{f}$ & 4 \\
3c & 4 & $3 \mathrm{~g}$ & 6 \\
\hline
\end{tabular}




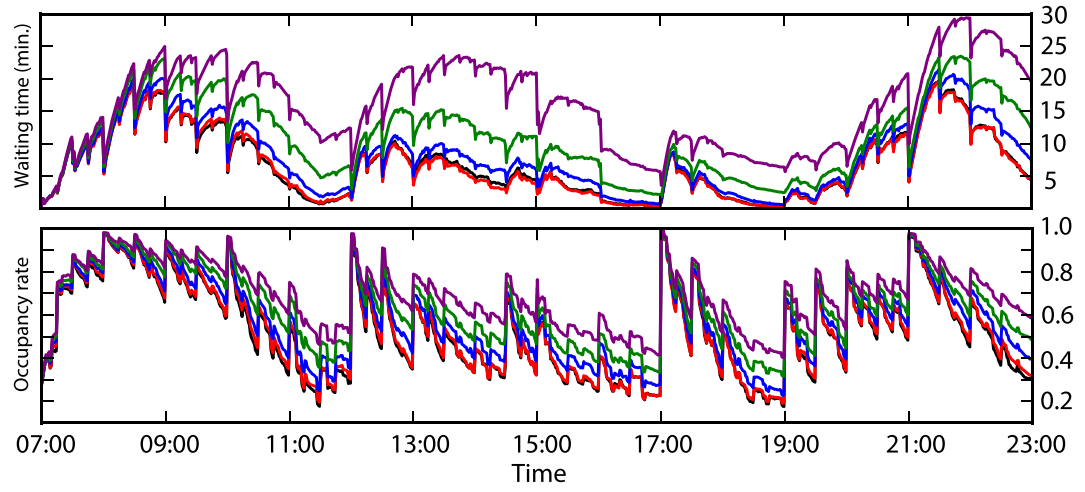

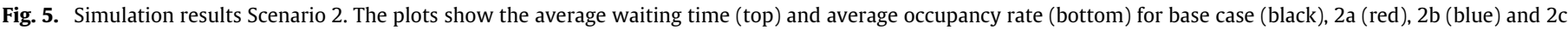
(green), 2d (purple). (For interpretation of the references to color in this figure legend, the reader is referred to the web version of this article.)

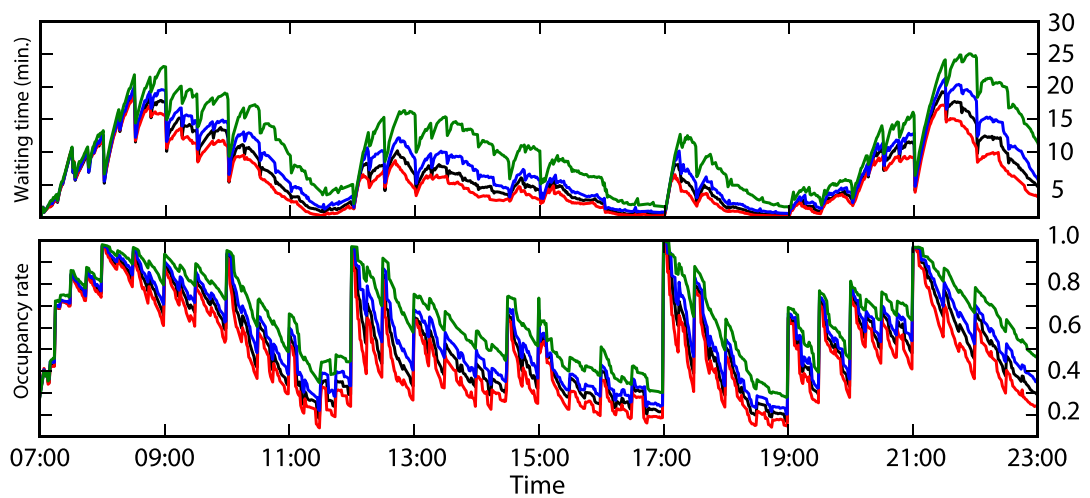

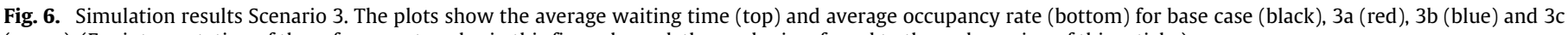
(green). (For interpretation of the references to color in this figure legend, the reader is referred to the web version of this article.)

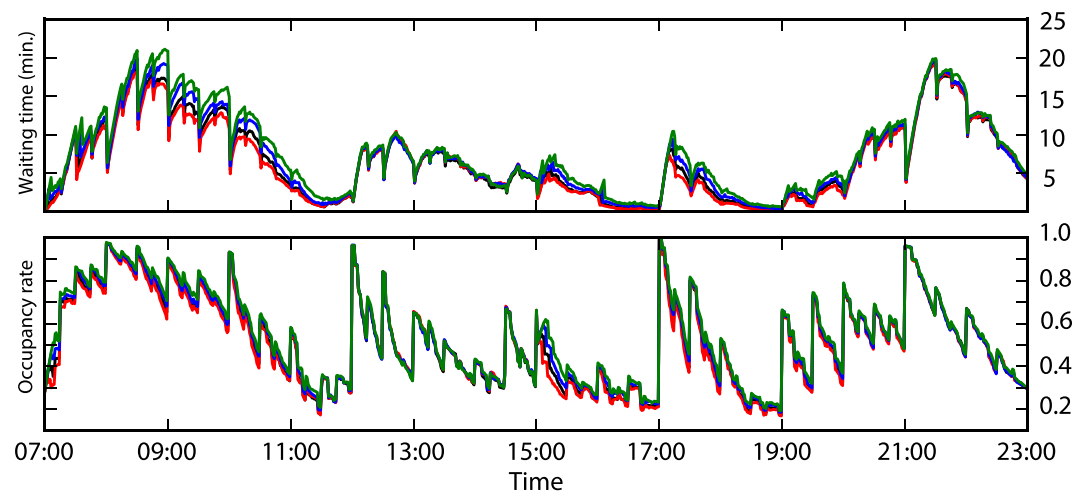

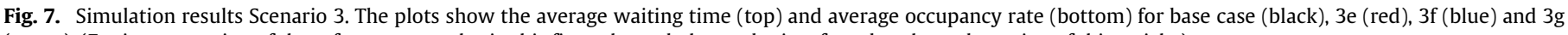
(green). (For interpretation of the references to color in this figure legend, the reader is referred to the web version of this article.)
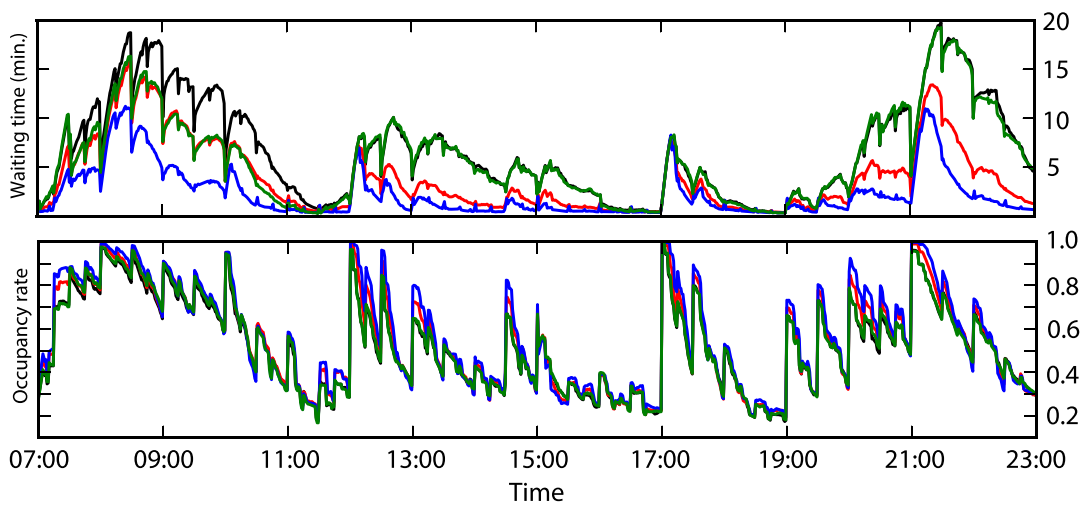

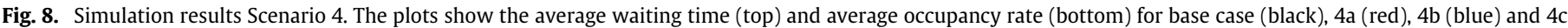
(green). (For interpretation of the references to color in this figure legend, the reader is referred to the web version of this article.) 
Table 8

Configurations Scenario 4.

\begin{tabular}{ll}
\hline Scenario & Allocation \\
\hline $4 \mathrm{a}$ & Fixed care workers provide support to two SSLFs. \\
$4 \mathrm{~b}$ & Fixed care workers provide support to all four SSLFs. \\
$4 \mathrm{c}$ & All flexible care workers provide support to all four SSLFs. \\
\hline
\end{tabular}

\subsection{Scenario 5: number of clients}

To assess the impact of more clients per SSLF, while keeping the number of hours per client roughly fixed, three alternative scenarios are examined. Tables 9 and 10 show the shifts for each scenario. The results (see Fig. 9 and Table A.13) show that a change in the number of clients per SSLF has a substantial impact on the performance. Reducing the number of clients per SSLF from 6 to 5 leads to a $50.44 \%$ increase in the total average waiting time. In addition, the top plot in Fig. 9 shows that reducing the number of clients from 6 to 5 results in average waiting times up to $40 \mathrm{~min}$ around 9:30 and 11:00 h. With 8 clients per SSLF the total average waiting drops below $5 \mathrm{~min}$ with a 15 min service level of almost $92 \%$. The maximum average waiting time drops to around $13 \mathrm{~min}$. We note that with more clients per SSLF, the flexibility significantly increases. Thus, the combination of economies of scale and increased flexibility greatly improves the performance of SSLFs.

\subsection{Scenario 6: allocation policy}

In Scenario 6a we examine the effect of organizing scheduled and unscheduled care separately. This approach allows to increase the demand-side flexibility of scheduled care by using time windows during which the activities are supposed to be carried out. More specifically, using the OERplanner algorithm, scheduled healthcare tasks are planned (if possible) within 15 min from the time preference of the client. In addition, for unscheduled healthcare care tasks, the FCFS approach is used. In this scenario, the supply of care and support is organized on a larger scale, i.e., care workers are assigned to multiple SSLFs. During day-time (7:00-23:00 h), four care workers are assigned to handle scheduled healthcare tasks according to a predetermined schedule. These predetermined schedules are also referred to as 'care routes'. In addition, between 7:00 and 23:00, two care workers are available for the provision of unscheduled care. These care workers are fully flexible as they can handle tasks for all of the four SSLFs.

Scenario 6a is compared with a FCFS approach in which, between 7:00 and 23:00, there are four fixed care workers. These care workers have the flexibility to handle tasks of two SSLFs. Additionally, there are two care workers available between 7:00 and 23:00. These care workers can handle tasks of all four SSLFs. This scenario is referred to as Scenario 6b. See Table A.14 for the configurations of both scenarios.

See Table 11 for the 15 min service level of the two scenarios under study. The results show that Scenario 6b outperforms Scenario 6a in terms of service level. This can be explained by the fact that the allocation flexibility is reduced when scheduled and unscheduled tasks are organized separately. However, the advantage of working with a predetermined schedule is that it provides a more clear structure and coordination in the provision of care and support.

\section{Service-level-based staffing}

In other service industries such as call-centers, staffing decisions are often based on minimizing staffing costs while maintaining an acceptable level of service, where level of service refers to the responsiveness to the customers' demand (see e.g., [32]). When it comes to call-center performance management,

Table 9

Configurations Scenario 5a and 5b.

\begin{tabular}{|c|c|c|c|c|}
\hline \multicolumn{5}{|l|}{$\begin{array}{l}\text { Scenario } 5 a \\
5 \text { clients }\end{array}$} \\
\hline Shift & Time hours & Allocation to \# of SSLFs & Number of shifts & Total hr per $24 \mathrm{hrs}$ \\
\hline $\begin{array}{l}\text { Day } \\
\text { Evening } \\
\text { Total } \\
\text { Hours/client }\end{array}$ & $\begin{array}{l}7: 00-15: 00 \\
15: 00-23: 00\end{array}$ & $\begin{array}{l}1 \\
1\end{array}$ & $\begin{array}{l}4 \\
4\end{array}$ & $\begin{array}{l}32 \\
32 \\
64 \\
3.20\end{array}$ \\
\hline \multicolumn{5}{|l|}{$\begin{array}{l}\text { Scenario 5b } \\
7 \text { clients }\end{array}$} \\
\hline Shift & Time hours & Allocation to \# of SSLFs & Number of shifts & Total per 24 hrs \\
\hline $\begin{array}{l}\text { Day } \\
\text { Evening } \\
\text { Morning assistance } \\
\text { Evening assistance } \\
\text { Total } \\
\text { Hours/client }\end{array}$ & $\begin{array}{l}7: 00-15: 00 \\
15: 00-23: 00 \\
7: 30-11: 30 \\
16: 00-20: 00\end{array}$ & $\begin{array}{l}1 \\
1 \\
2 \\
4\end{array}$ & $\begin{array}{l}4 \\
4 \\
4 \\
2\end{array}$ & $\begin{array}{l}32 \\
32 \\
16 \\
8 \\
88 \\
3.14\end{array}$ \\
\hline
\end{tabular}

Table 10

Configurations Scenario 5c.

\begin{tabular}{|c|c|c|c|c|}
\hline \multicolumn{5}{|l|}{$\begin{array}{l}\text { Scenario } 5 c \\
8 \text { clients }\end{array}$} \\
\hline Shift & Time hours & Allocation to \# of SSLFs & Number of shifts & Total per $24 \mathrm{hrs}$ \\
\hline Day & $7: 00-15: 00$ & 1 & 4 & 32 \\
\hline Evening & $15: 00-23: 00$ & 1 & 4 & 32 \\
\hline Morning assistance & $7: 30-11: 30$ & 2 & 4 & 16 \\
\hline Morning assistance & $7: 30-11: 30$ & 4 & 1 & 4 \\
\hline Evening assistance & $16: 00-20: 00$ & 4 & 2 & 8 \\
\hline Evening assistance & $19: 00-23: 00$ & 4 & 2 & 8 \\
\hline Total & & & & 100 \\
\hline Hours/client & & & & 3.13 \\
\hline
\end{tabular}



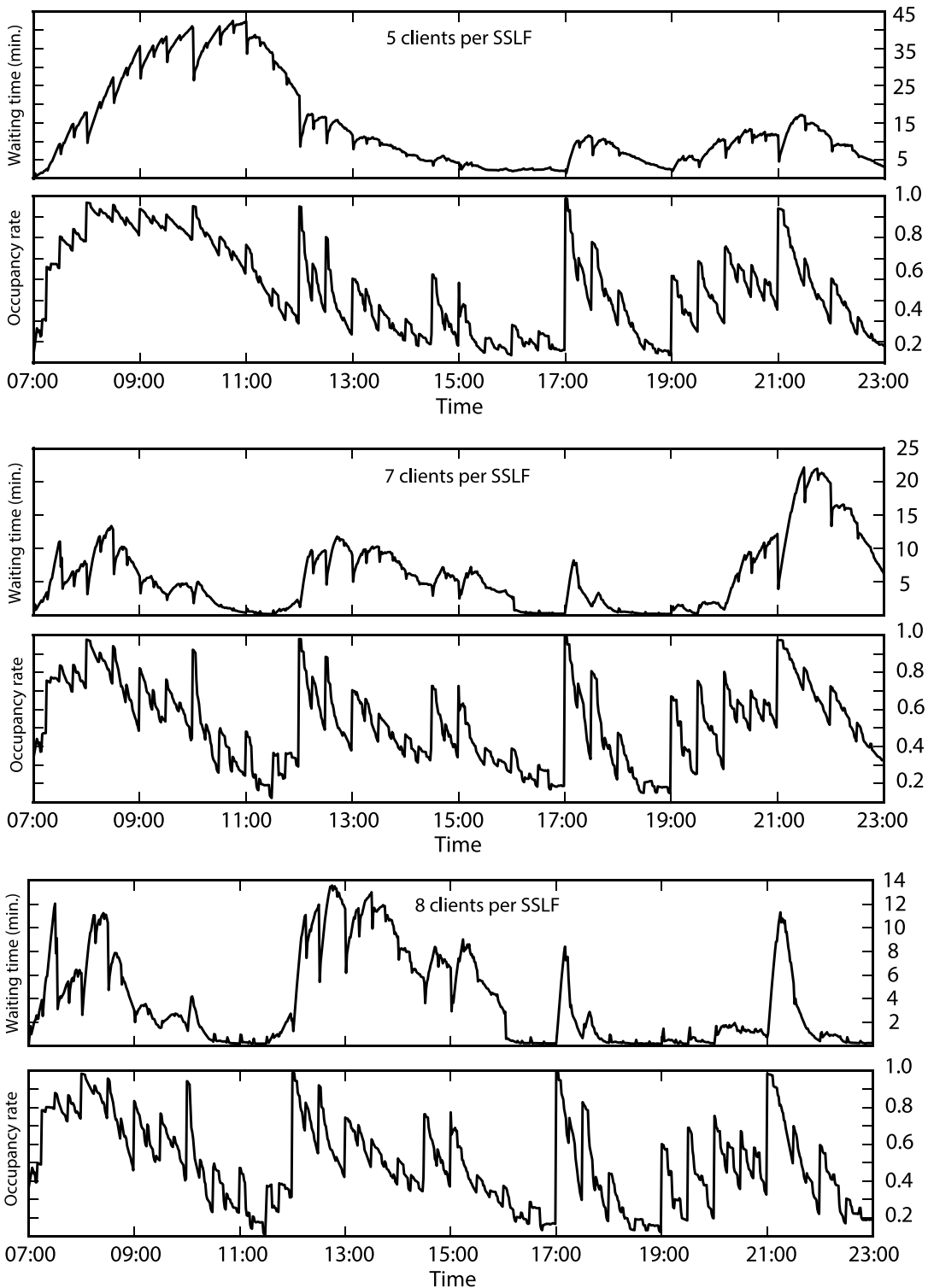

Fig. 9. Simulation results Scenario 5: The plots show the average waiting time and average occupancy rate during the day.

service level is often defined as the percentage of calls $(X \%)$ answered within $Y$ seconds. In this section we use the following context specific definition: the \% of care request in time-interval $\Delta t$ for which a care worker was present with the client within 15 min. Although, in the base case the overall service level is $88.35 \%$, the average service level fluctuates considerably during the course of the day (see Fig. 10). Around 7:30-8:00 and 20:30-21:00 h a serious drop in the average service level can be observed. This example shows that basing staffing decisions on averages without taking variability into account can be dangerous, something which is also referred to as "the flaw of averages" [33]. To maintain an acceptable level of service throughout the day, call-centers often base their measurements and staffing decisions on half-hour intervals.

In line with this reasoning, we consider the problem of minimizing the total number of care workers, while maintaining an acceptable level of service throughout the day. The average service level goal is set at $95 / 15$. Hence, for $95 \%$ of the care request in each half-hour time-interval a care worker should be with the client within $15 \mathrm{~min}$. Furthermore, we assume each of the care workers to be fully flexible. That is, they can handle tasks for all of the four SSLFs. We use the simulation tool to determine how many care
Table 11

Results Scenario 6.

\begin{tabular}{llll}
\hline \multirow{2}{*}{ Scenario } & \multicolumn{3}{l}{15 min service level } \\
\cline { 2 - 4 } & Overall & Scheduled care & Unscheduled care \\
\hline Base case & $88.36 \%$ & - & - \\
6a & $90.79 \%$ & $89.59 \%$ & $91.75 \%$ \\
6b & $96.48 \%$ & - & - \\
\hline
\end{tabular}

workers are needed in each time-interval to obtain the required service level. Starting at the first time-interval (7:00-7:30) we determine the required number of care workers and step by step include the subsequent time interval.

Fig. 11 shows the average service level during the day when applying service-level based staffing. The overall average service level and total average occupancy rate under this staffing policy are $96.38 \%$ and $61.18 \%$ respectively. On the top side of the $x$-axis, for each half-hour interval, the required number of care workers is compared with the base case. A total of 59 working hours are needed to achieve a 95/15 service level in each half-hour interval, which is $22 \%$ less than in the base case. The reasons for this are (1) the increased allocation flexibility of the care workers (see also 


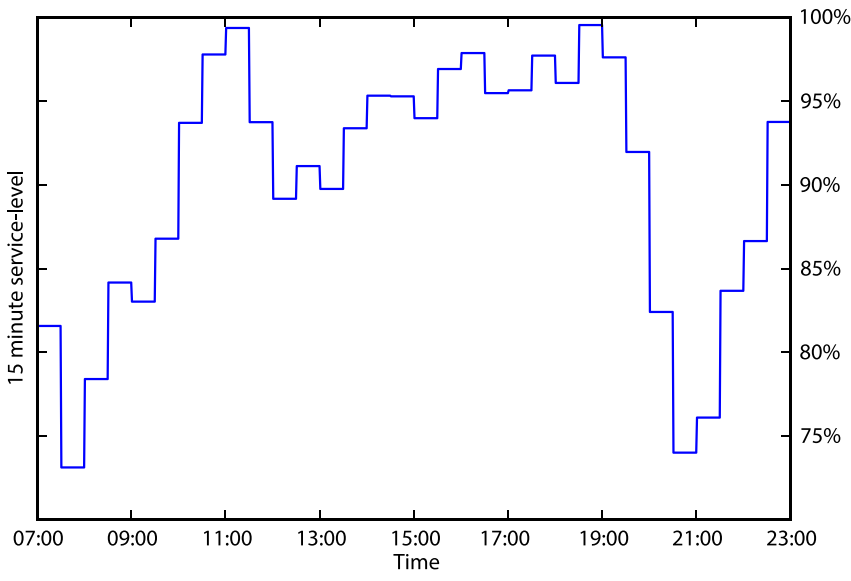

Fig. 10. Base case: 15 min service level during the day.

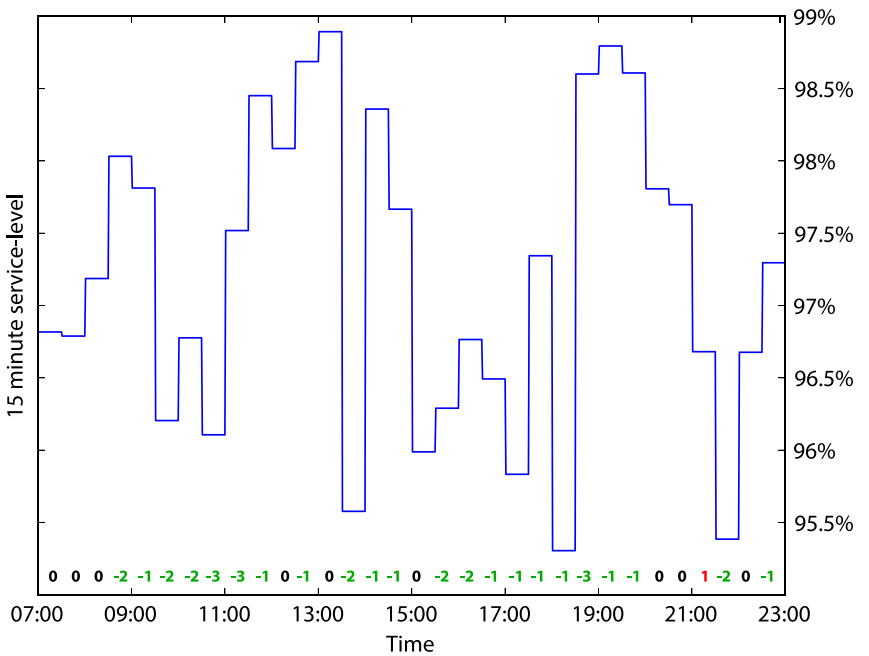

Fig. 11. Service-level based staffing policy: 15 min service level during the day.

Scenario 4) and (2) a reduction of overstaffing during the less busy periods of the day.

\section{Conclusions and discussion}

In this study we analyzed the performance of SSLFs, focusing on the problem of meeting the time preferences of residents regarding the delivery of care and support. The case of SSLFs is interesting as small-scale care is under increasing financial pressure due to budget cuts. A simulation model is developed which resembles the current care delivery process of a Dutch nursing home department with four SSLFs. The model is used to examine the performance under various scenarios. We modeled scheduled care using historical data and unscheduled care using a Poisson-Gamma mixture model.

With a total average waiting time of $5.73 \mathrm{~min}$ and a $15 \mathrm{~min}$ service level of $88.35 \%$, the overall performance under the base case is beyond our expectations. Nonetheless, (1) the average waiting time fluctuates considerably over the course of a day, with average waiting times up to 20 min around 8:30 and 22:00 h. In addition, with $52.81 \%$, the occupancy rate is relatively low. When it comes to the arrival process, the overall performance seems relatively insensitive to an increase in the average arrival rate of unscheduled care. In addition, the performance under time-homogeneous Poisson arrivals differs only slightly from the performance under Poisson-Gamma mixture assumption. With regard to the average care duration, the results show that, the overall performance is relative sensitive to changes. This is an interesting finding as under current Dutch healthcare reforms, the care needs of nursing home clients are likely to become more severe. The results also indicate, that an increase in allocation flexibility of fixed care workers has a substantial positive effect on the overall performance, which is in line with the findings presented in [23]. As such, when meeting the time preferences of clients is considered to be an important QoL factor, the SSLF principle of working with a small fixed team of care workers is open to question. A change in the number of clients per SSLF under constant average costs also has a substantial impact on the performance. A reduction in the number of clients per SSLF from 6 to 5 leads to a $50.44 \%$ increase in the total average waiting time. With 8 clients per SSLF the total average waiting drops below $5 \mathrm{~min}$ with a 15 min service level of almost 92\%. In the last scenario we examined the effect of organizing scheduled and unscheduled care separately, whereas dedicated workers are assigned to handle scheduled healthcare tasks according to a predetermined schedule. The results of this scenario show that it does not lead to better performance in terms of service level compared to a fully FCFS policy, in which no distinction is made between scheduled and unscheduled healthcare tasks. The main reason for this is that the allocation flexibility is reduced when scheduled and unscheduled tasks are organized separately. However, making all care workers fully flexible would be hard to accomplish in practice. It would most likely lead to a lack of overview and stressful situations. Hence, an important advantage of working with a predetermined schedule for unscheduled care is that it provides a more clear structure and coordination in the provision of care and support.

In Section 6 we consider the problem of minimizing the total number of care workers, while maintaining a 95/15 service level throughout the day. For each half-hour interval we determined how many care workers are needed to obtain the required service level. We did this under the assumption that care workers

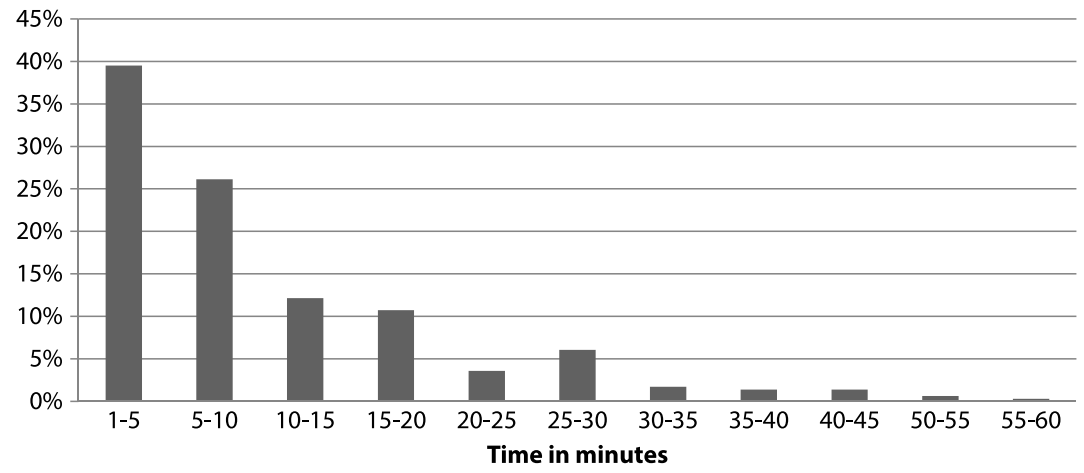

Fig. A.12. Distribution of care delivery times for scheduled care. 

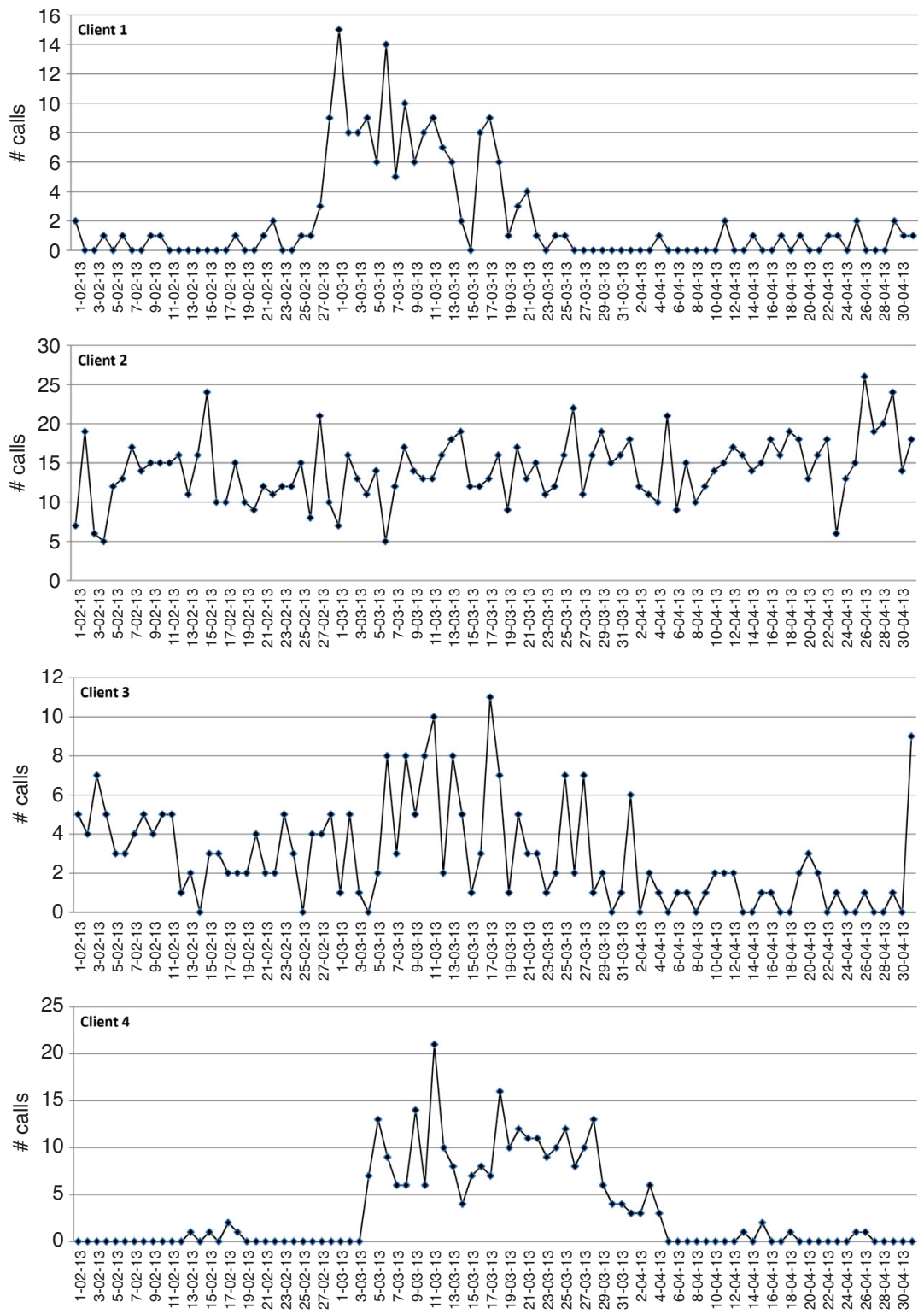

Fig. A.13. Number of calls during the day for client $1,2,3$ and 4.
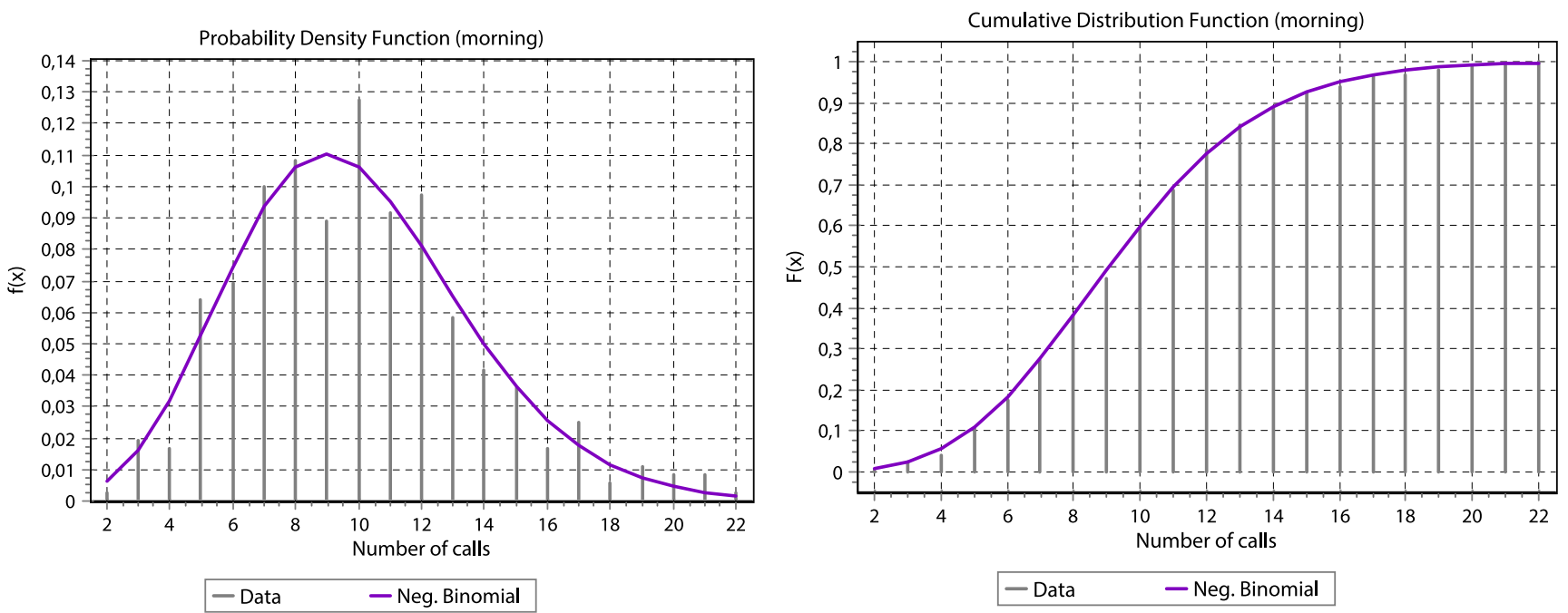

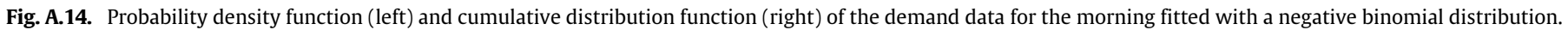


Table A.12

Main characteristics dataset scheduled care.

\begin{tabular}{llcc}
\hline Dep. & Type of care & Number of clients & Average \# rows \\
\hline A & Somatic care (assisted living) & 49 & 123 \\
B & Psychogeriatric care (assisted living) & 9 & 61 \\
C & Somatic care & 34 & 176 \\
D & Somatic care & 28 & 260 \\
E & Psychogeriatric care & 33 & 263 \\
Total & & 153 & 884 \\
\hline
\end{tabular}
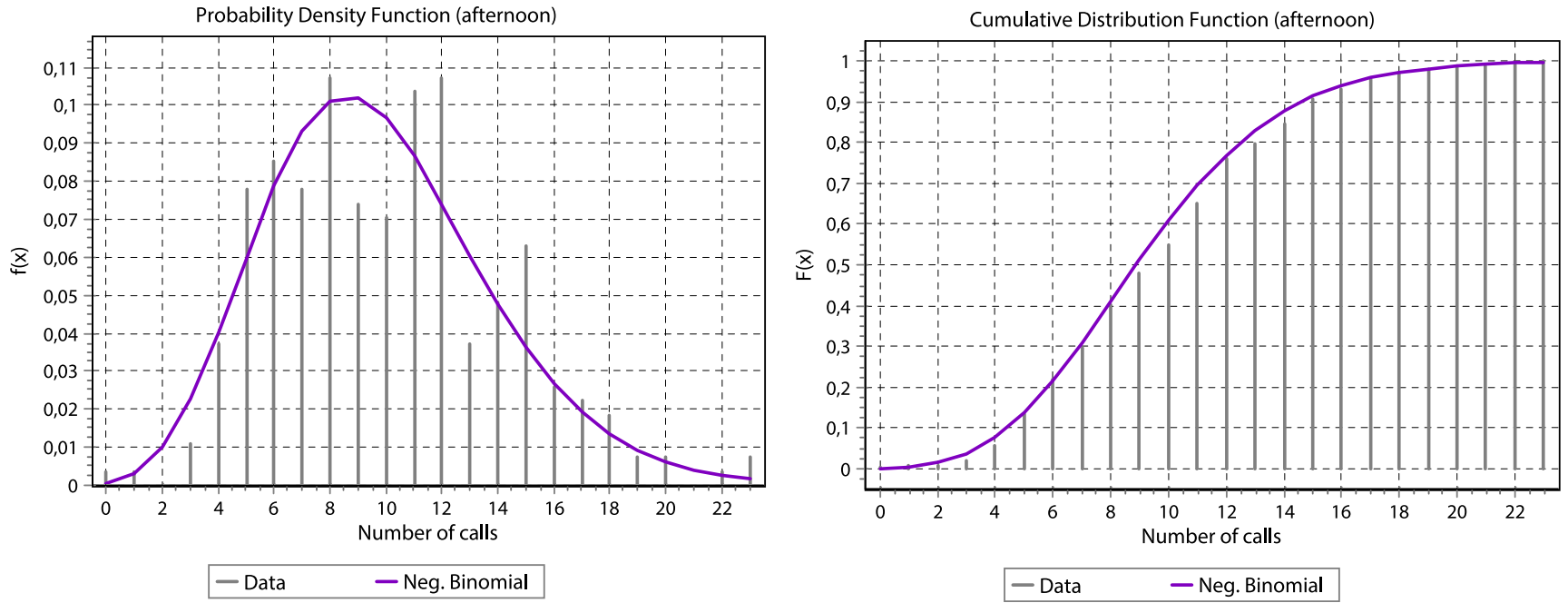

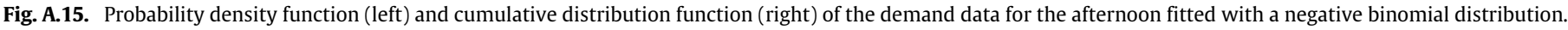
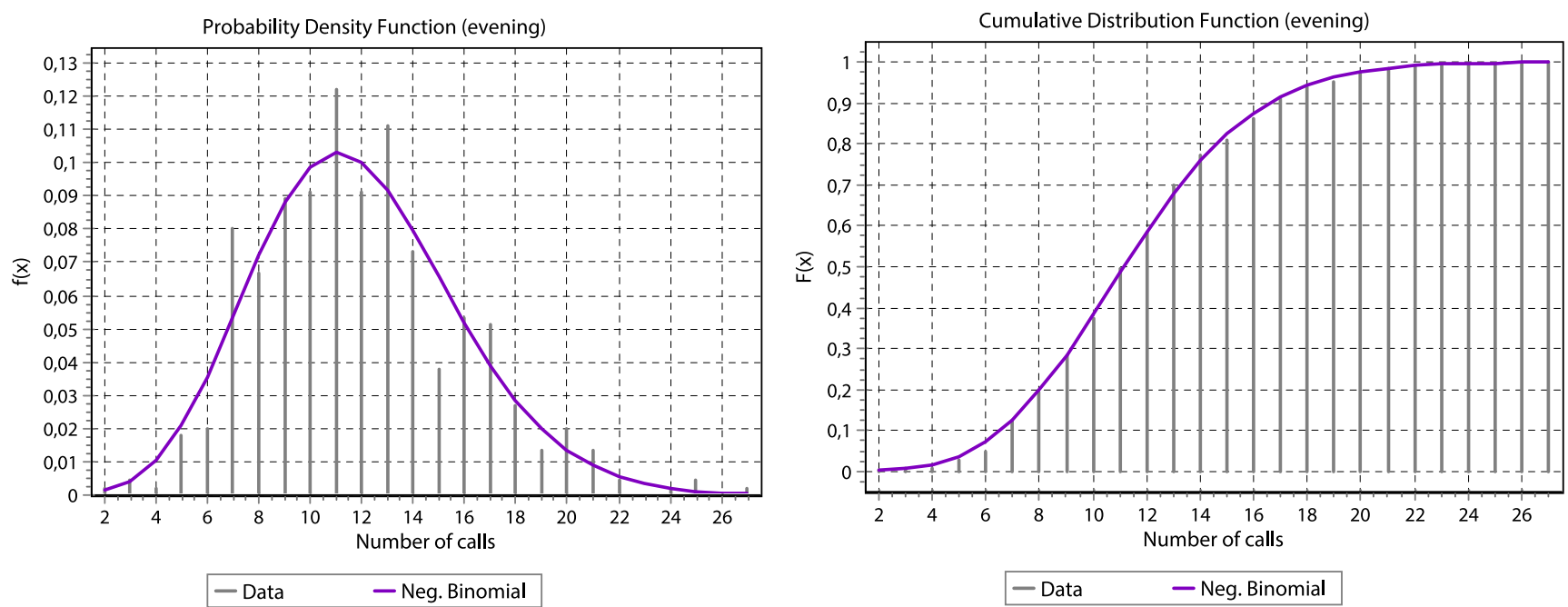

Fig. A.16. Probability density function (left) and cumulative distribution function (right) of the demand data for the evening fitted with a negative binomial distribution.

can handle tasks for all of the four SSLFs. The results show that allocation flexibility together with numerical flexibility has a substantial positive effect on the performance. In practice however, a service-level-based staffing approach is not fully applicable. For example, the collective labor agreement restricts the way in which personnel can be deployed. Nevertheless, we do see there is considerable scope for improvement when it comes to increasing flexibility. In this respect, nursing homes could learn from other service industries.

In conclusion, we can say that the shifts presented in the base case provide a good point of departure. In addition, to further improve the performance the focus should lie on: (1) increasing the allocation flexibility of care workers and the number of clients per SSLF, and (2) time dependent staffing.

This study also shows that simulation is useful for assessing and improving daily nursing home operations. The presented simulation model is an important step to better understand the real-life performance of SSLFs and the underlying relationships involved. It provides a basis for building a decision support tool for nursing home managers. An important next step is building an interface to allow nursing home managers to use the simulation tool in daily practice. 
Table A.13

Overall performance Scenarios 1-5.

\begin{tabular}{llll}
\hline Scenario & 15 min service level & Total average waiting time $(\min )$ & Total average occupancy rate \\
\hline Base case & $88.35 \%$ & 5.73 & $52.81 \%$ \\
1a & $88.99 \%$ & 5.47 & $46.60 \%$ \\
1b & $87.31 \%$ & 6.20 & $58.53 \%$ \\
1c & $84.96 \%$ & 7.27 & $69.37 \%$ \\
1d & $89.16 \%$ & 5.36 & $48.23 \%$ \\
1e & $88.74 \%$ & 5.56 & $54.65 \%$ \\
1f & $87.95 \%$ & 5.90 & $60.60 \%$ \\
1g & $85.67 \%$ & 6.92 & $71.95 \%$ \\
2a & $88.04 \%$ & 5.87 & $53.60 \%$ \\
2b & $85.84 \%$ & 6.76 & $57.78 \%$ \\
2c & $81.85 \%$ & 8.68 & $63.09 \%$ \\
2d & $77.69 \%$ & 11.54 & $69.26 \%$ \\
3a & $90.56 \%$ & 4.27 & $47.53 \%$ \\
3b & $85.68 \%$ & 7.17 & $56.84 \%$ \\
3c & $77.67 \%$ & 10.31 & $65.06 \%$ \\
3e & $89.20 \%$ & 5.33 & $51.29 \%$ \\
3f & $87.53 \%$ & 6.08 & $53.36 \%$ \\
3g & $86.60 \%$ & 6.51 & $54.61 \%$ \\
4a & $91.57 \%$ & 4.83 & $55.02 \%$ \\
4b & $94.29 \%$ & 4.10 & $56.78 \%$ \\
4c & $88.67 \%$ & 5.52 & $52.78 \%$ \\
5a & $84.28 \%$ & 8.62 & $55.41 \%$ \\
5b & $89.98 \%$ & 5.19 & $51.57 \%$ \\
5c & $92.88 \%$ & 4.15 & $49.29 \%$ \\
\hline
\end{tabular}

Table A.14

Configurations Scenario 6.

\begin{tabular}{lllll}
\hline Shift & Time hours & Allocation to \# of SSLFs & Number of shifts & Total per 24 hrs \\
\hline Scenario 6a & & & & \\
\hline Sched. care day & $7: 00-15: 00$ & Care routes & 4 & 32 \\
Sched. care evening & $15: 00-23: 00$ & Care routes & 4 & 32 \\
Unsched. care morning & $7: 00-11: 00$ & 4 & 2 & 16 \\
Unsched. care afternoon & $11: 00-19: 00$ & 4 & 2 & 8 \\
Unsched. care evening & $19: 00-23: 00$ & 4 & 2 & 96 \\
Total & & & & 4.00 \\
Hours/client & & & 4 & 32 \\
\hline Scenario 6b & & 4 & 32 \\
\hline Day & & & 2 & 8 \\
Evening & $7: 00-15: 00$ & 2 & 2 & 8 \\
Morning assistance & $15: 00-23: 00$ & 2 & 2 & 96 \\
Afternoon assistance & $7: 00-11: 00$ & 4 & & 4.00 \\
Evening assistance & $11: 00-19: 00$ & 4 & & \\
Total & $19: 00-23: 00$ & 4 & & \\
Hours/client & & & & \\
\hline
\end{tabular}

\section{Appendix}

See Tables A.12-A.14 and Figs. A.12-A.16.

\section{References}

[1] H. Verbeek, Redesigning Dementia Care: An Evaluation of Small-Scale Homelike Care Environments, Maastricht University, 2011.

[2] B. Willemse, M. Depla, D. Smit, A. Pot, The relationship between smallscale nursing home care for people with dementia and staff's perceived job characteristics, Int. Psychogeriatr. 26 (5) (2014) 805-816.

[3] A.H. De Rooij, K.G. Luijkx, J. Schaafsma, A.G. Declercq, P.M. Emmerink, J.M. Schols, Quality of life of residents with dementia in traditional versus small scale long-term care settings: a quasi-experimental study, Int. J. Nurs. Stud. 49 (8) (2012) 931-940.

[4] H. Verbeek, S.M. Zwakhalen, E. van Rossum, T. Ambergen, G.I. Kempen, J.P. Hamers, Effects of small-scale, home-like facilities in dementia care on residents behavior, and use of physical restraints and psychotropic drugs: a quasi-experimental study, Int. Psychogeriatr. 26 (4) (2014) 657-668.

[5] E. Hamrock, J. Parks, J. Scheulen, F.J. Bradbury, Discrete event simulation for healthcare organizations: a tool for decision making, J. Healthcare Manag. 58 (2) (2013) 110-125

[6] S. Brailsford, J. Vissers, OR in healthcare: a European perspective, European J. Oper. Res. 212 (2) (2011) 223-234.
[7] M.M. Günal, M. Pidd, Discrete event simulation for performance modelling in health care: a review of the literature, J. Simul. 4 (1) (2010) 42-51.

8] D. Fone, S. Hollinghurst, M. Temple, A. Round, N. Lester, A. Weightman, et al., Systematic review of the use and value of computer simulation modelling in population health and health care delivery, J. Public Health 25 (4) (2003) 325-335.

[9] H. Saadouli, B. Jerbi, A. Dammak, L. Masmoudi, A. Bouaziz, A stochastic optimization and simulation approach for scheduling operating rooms and recovery beds in an orthopedic surgery department, Comput. Ind. Eng. 80 (2015) 72-79.

[10] Z. Zhang, X. Xie, Simulation-based optimization for surgery appointment scheduling of multiple operating rooms, IIE Trans. 47 (9) (2015) 998-1012.

[11] R. Konrad, K. DeSotto, A. Grocela, P. McAuley, J. Wang, J. Lyons, et al., Modeling the impact of changing patient flow processes in an emergency department: insights from a computer simulation study, Oper. Res. Health Care 2 (4) (2013) 66-74.

[12] Y.H. Kuo, Integrating simulation with simulated annealing for scheduling physicians in an understaffed emergency department, HKIE Trans. 21 (4) (2014) 253-261.

[13] Z. Zhu, B. Hoon Hen, K. Liang Teow, Estimating ICU bed capacity using discrete event simulation, Int. J. Health Care Qual. Assur. 25 (2) (2012) 134-144.

[14] J.D. Griffiths, M. Jones, M.S. Read, J.E. Williams, A simulation model of bedoccupancy in a critical care unit, J. Simul. 4 (1) (2010) 52-59.

[15] K. Van Eeden, D. Moeke, R. Bekker, Care on demand in nursing homes: a queueing theoretic approach, Health Care Manage. Sci. (2014) [Advance online publication] 
[16] E. Thackery, M. Harris, The Gale Encyclopedia of Mental Disorders: AL. Vol. 1, Gale Group, 2003.

[17] J. De Lange, Omgaan met dementie [Dealing with dementia]: het effect van geïntegreerde belevingsgerichte zorg op adaptatie en coping van mensen met dementie in verpleeghuizen; een kwalitatief onderzoek binnen een gerandomiseerd experiment. Erasmus MC: University Medical Center, Rotterdam; 2004.

[18] E. Finnema, R. Dröes, M. Ribbe, W. Van Tilburg, The effects of emotion-oriented approaches in the care for persons suffering from dementia: a review of the literature, Int. J. Geriatr. Psychiatry 15 (2) (2000) 141-161.

[19] R. Dröes, L. Van Mierlo, H. Van der Roest, F. Meiland, Focus and effectiveness of psychosocial interventions for people with dementia in institutional care settings from the perspective of coping with the disease, Non-Pharmacological Therapies Dementia 1 (2) (2010) 139-161.

[20] D. Smit, J. De Lange, B. Willemse, A.M. Pot, The relationship between small-scale care and activity involvement of residents with dementia, Int. Psychogeriatr. 24 (5) (2012) 722-732.

[21] S. Te Boekhorst, M.F.I.A. Depla, J. de Lange, A.M. Pot, J.A. Eefsting, The effects of group living homes on older people with dementia: a comparison with traditional nursing home care, Int. J. Geriatr. Psychiatry 24 (9) (2009) 970-978. Available from: http://dx.doi.org/10.1002/gps.2205.

[22] H. Verbeek, S.M. Zwakhalen, E. van Rossum, T. Ambergen, G.I. Kempen, J.P. Hamers, Dementia care redesigned: effects of small-scale living facilities on residents, their family caregivers, and staff, J. Am. Med. Dir. Assoc. 11 (9) (2010) 662-670.
[23] D. Moeke, G. Koole, L. Verkooijen, Scale and skill-mix efficiencies in nursing home staffing: inside the black box, Health Syst. 3 (1) (2014) 18-28.

[24] A. Lieder, D. Moeke, G.M. Koole, R. Stolletz, Task scheduling in long-term care facilities: a client-centered approach, Oper. Res. Health Care (2015) [Advance online publication]

[25] D. Moeke, R. Bekker, B. Schmidt, Ebbs and flows in daily nursing home operations: a demand and workload analysis. Working paper. 2015;Available from: http://ssrn.com/abstract=2780574.

[26] K. Pearson, On the criterion that a given system of deviations from the probable in the case of a correlated system of variables is such that it can be reasonably supposed to have arisen from random sampling, Lond. Edinb. Dublin Philos. Mag. J. Sci. 50 (302) (1900) 157-175.

[27] G. Jongbloed, G. Koole, Managing uncertainty in call centres using Poisson mixtures, Appl. Stoch. Models Bus. Ind. 17 (4) (2001) 307-318.

[28] A.C. Cameron, P.K. Trivedi, Regression Analysis of Count Data. Vol. 53, Cambridge University Press, Cambridge, 2013.

[29] F.J. Massey Jr., The Kolmogorov-Smirnov test for goodness of fit, J. Amer. Statist. Assoc. 46 (253) (1951) 68-78.

[30] (NZa) TDHA. Bekostiging ADL-assistentie [Financing ADL-assistance]. Utrecht: $\mathrm{NZa} ; 2013$.

[31] S. Robinson, Simulation: The Practice of Model Development and Use, Palgrave Macmillan, London, 2014.

[32] G. Koole, A. Mandelbaum, Queueing models of call centers: An introduction, Ann. Oper. Res. 113 (1) (2002) 41-59.

[33] S. Savage, The flaw of averages, Harv. Bus. Rev. 80 (11) (2002) 20-21. 